\title{
Inter disciplinary approach of chronic periapical lesion with extraoral sinus tract opening on to the nasal floor: A case report
}

\author{
Sivarajan Ranjithkumar ${ }^{1 *}$, Jogikalmat Krithika Datta ${ }^{2}$, Natanasabhapathy Velmurugan ${ }^{3}$, Sreekumar Kalavichirattil ${ }^{4}$ \\ ${ }^{1}$ Reader, ${ }^{2,4}$ Associate Professor, ${ }^{3}$ Professor and HOD, ${ }^{1-3}$ Dept. of Conservative Dentistry and Endodontics, ${ }^{4}$ Dept. of Oral and Maxillofacial \\ Surgery, ${ }^{2-4}$ Faculty of Dentistry, ${ }^{1}$ SRM Institute of Science and Technology Kattankulathur, Kanchipuram, Tamil Nadu, ${ }^{2-4}$ Meenakshi \\ Academy of Higher Education and Research, Chennai, Tamil Nadu, India
}

\begin{abstract}
Dental pulp and periodontium are closely related and the pathway of communication between these structures helps in determining the progress and prognosis of the disease. Though periapical lesions can be managed non-surgically, surgical intervention is needed in cases of recalcitrant chronic periapical lesions that do not respond to conventional endodontic therapy. This case report highlights the surgical management of chronic periapical lesion of endodontic origin with an unusual location of the extra-oral sinus on the nasal floor.
\end{abstract}

Keywords: Chronic periapical lesion, Nasal floor, Extra-oral sinus tract, Deformed nose, Surgical endodontics.

\section{Introduction}

Endodontic treatment is aimed to eliminate bacteria from infected root canals that will be later sealed to prevent bacterial recontamination. Elimination of the bacterial stimuli is said to resolve the periapical lesion. If the irritants or the bacterial byproducts persist in the canal, it may lead to chronic periapical lesion. The most common cause of sinus tract is a chronic periapical abscess. ${ }^{1}$ The periapical inflammatory process results from a necrotic pulp and spreads into the surrounding periodontal ligament and bone. Following the onset of apical periodontitis, depending on the inflammatory process and immunological response, bone resorption is induced. The inflammation then spreads peripherally through least path of resistance until the cortex of the bone is reached. A subperiosteal abscess is formed following destruction of the cortical bone. ${ }^{2}$ In order to establish drainage, the periosteum is pierced and depending on factors like gravity and most importantly anatomic arrangement of adjacent muscles and fasciae, either an extra-oral or an intra-oral sinus tract is formed. ${ }^{3}$

Non-surgical management is the preferred treatment option in cases with sinus tract and cutaneous lesion. The elimination of the primary cause of endodontic infection results in resolution of the sinus tract between 5 to 14 days. ${ }^{4}$ This healing often occurs by secondary intention in majority of cases. Surgical intervention, if required is mostly for the non-healing lesions, esthetic correction of the cutaneous retraction or dimpling. 5

The following case report is the description of an extraoral sinus tract opening on to the nasal floor, draining a chronic periapical lesion of the left maxillary central incisor.

\section{Case Report}

A 32-year old male patient reported to the Department of Laryngology with the chief complaint of bleeding from the nose. Clinical examination was non-contributory to medical origin. The patient was referred to the Department of Conservative Dentistry \& Endodontics with a provisional diagnosis of focal infection of dental origin.

Past dental history revealed trauma to the upper front tooth 20 years before for which root canal therapy was initiated 2 years back for the same tooth. Extra oral examination revealed a deformed nose [Fig. 1(a)]. According to the patient the reason for nasal deformity was a result of infection during his childhood. Examination of the left nostril revealed presence of sinus opening on the anterior part of the nasal floor [Fig. 1(b)]. Intra oral examination revealed loss of coronal seal in access prepared left upper central incisor. Sinus tract tracing was done using a 30-size gutta-percha point (Dentsply Gutta Percha Points Dentsply Maillefer, India), which confirmed the presence of a sinus tract leading to the root apex of tooth \# 9. Further investigation showed the presence of a periapical radiolucency measuring $2 \times 2 \mathrm{~cm}$ involving tooth \#9 and the radiograph also confirmed access preparation [Fig. 1(c)]. Computerized Tomography (CT) scan taken previously for surgical correction of the nasal deformity also confirmed the presence of oro-nasal communication, Hence a Cone Beam Computerized Tomography(CBCT) scan was not considered for the diagnosis.

A diagnosis of previously initiated therapy with Chronic apical abscess associated sinus tract in \# 9 was made based on the clinical and radiographic examination.

Informed consent was obtained prior to initiation of treatment. Under rubber dam isolation access opening was modified to obtain straight-line access. Working length was determined using an apex locator (Root Zx, J Morita mfg. Corp, kyoto, JA) and later confirmed using a radiograph. Cleaning and shaping was done using k-files (Dentsply Maillefer, Colorinox, India) to master apical file size \# 70.

\footnotetext{
*Corresponding Author: Sivarajan Ranjithkumar, Dept. of Conservative Dentistry and Endodontics, SRM Institute of Science and Technology Kattankulathur, Kanchipuram, Tamil Nadu, India

Email: sranji09@gmail.com

http://doi.org/10.18231/j.ijce.2019.026
} 


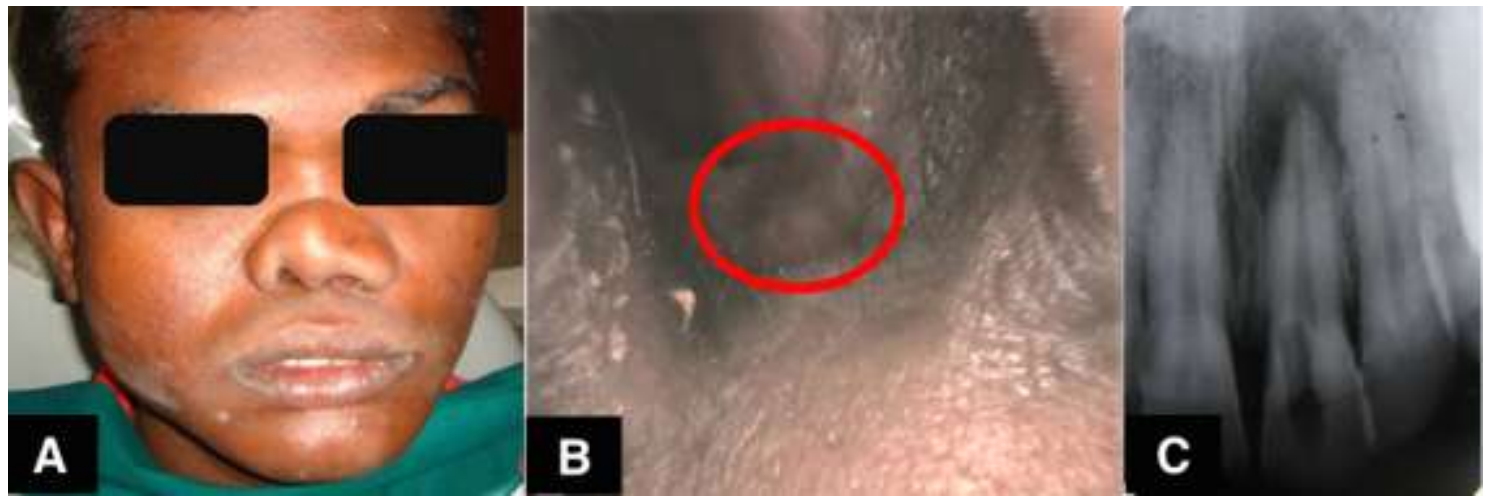

Fig. 1(a): Extra-oral examination revealed the presence of congenitally deformed nose. (b): Presence of sinus opening in the nasal floor. (c): Pre-operative Intra Oral Periapical Radiograph (IOPA)

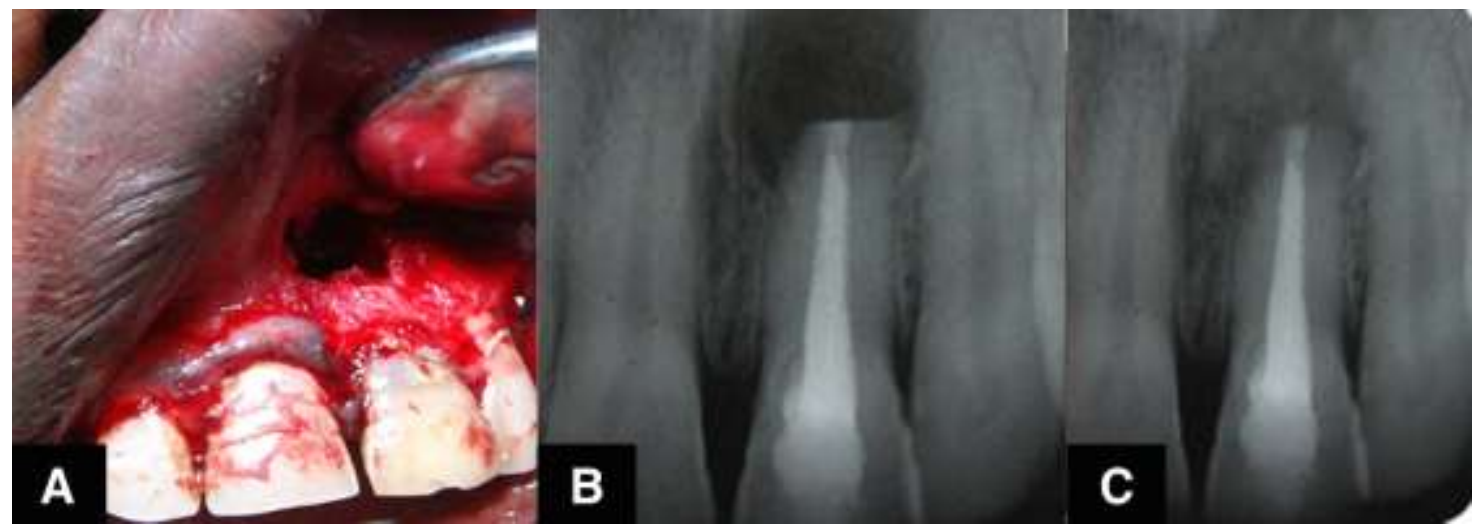

Fig. 2(a): Periapical lesion was curetted. 2(b): Retrograde filling done using IRM. (c): 18 month follow-up radiograph revealed satisfactory healing

Calcium hydroxide intracanal medicament (RC CAL, Prime dental products, Thane, India) was placed and access cavity was sealed with Cavit G(3M ESPE Deutschland GmbH, Neuss - Germany). Patient was recalled after a week for further treatment. However, patient reported the next day with a complaint of persistent discharge from the sinus tract. Examination of the sinus tract revealed sero-sanguinous discharge from the sinus. Since the root end was in close approximation to the nasal floor, patient was referred to department of oral and maxillofacial surgery for opinion. The recommendations of the oral surgeon were considered for a final treatment plan and the patient was advised for periapical surgery in relation to tooth $\# 9$ along with deepithelization of the sinus tract.

The canal was then irrigated with saline and 2\% chlorhexidine (Anabond Asep-RC, Stedman Pharma (P) Ltd., Chennai, Tamil Nadu) calcium hydroxide intracanal medicament was placed and temporized. Patient was recalled after a week for further treatment. The symptoms did not subside through the week that strengthened the surgical management plan.

On the surgical visit, obturation was done using guttapercha (Dentsply Maillefer, India) and AH plus sealer (AH Plus Root canal sealer, Dentsply DeTrey GmbH, Konstanz, Germany) by cold lateral compaction technique. Local anesthesia was achieved using $2 \%$ lignocaine with $1: 80,000$ epinephrine (Lignox 2\% A, Indoco Remedies Ltd, Mumbai, India). A full thickness rectangular mucoperiosteal flap was elevated in relation to tooth \#9 [Fig. 2(a)]. The presence of the lesion had resulted in denudation of the root apex labially. Under the dental operating microscope (Seiler precision microscope, St.Louis, Missouri), periapical lesion was curetted using surgical curette (Hu-friedy manufacturing company, Inc, Chicago, Illinosis, USA) and the specimens were sent for biopsy.

Root end resection was performed using a slow speed micromotor (\# 702 SS White burs Inc, Lakewood, NJ, USA). Following retropreparation using ultrasonic tips (Satelec, Shangai, China), retrograde filling was done with IRM (Dentsply, IRM Ivory ZOE Intermediate Restorative Material, Dentsply Maillefer, India) [Fig. 2(b)]. Thorough debridement of the periapical region was done using sterile saline. Following periapical surgery de-epithelization of the sinus tract was done. The sinus tract was traced from the periapical region to the nasal floor and the epithelial lining of the sinus was removed by carefully curetting the sinus wall. The flap was repositioned and approximated using a 30 Non-absorbable silk sutures (Ethicon, Johnson \& Johnson Pvt.Ltd, Himachal pradesh, India). Sinus opening present in the nasal floor was sutured using a 6-0 resorbable vicryl sutures (3M, United States Suture Company (USSC), USA).

Post operative instructions were given to the patient. Biopsy report confirmed the lesion to be infected dental 
cyst. The patient was reviewed after 4 days and suture removal was done. The outcome was favorable with healing of the sinus tract and complete absence of symptoms. The patient was followed for 18 months throughout which he remained asymptomatic [Fig. 2(c)].

\section{Discussion}

Sinus tract is an asymmetrical type of drainage pathway from a deep focus of chronic infection through tissue and/or bone to an opening on the surface. Odontogenic infection like any other infection in the body, follow the path of least resistance. ${ }^{6}$ Chronic abscess occurring as a result of pulpal necrosis remains confined in a circumscribed area leading to collection of pus and certain degree of bone destruction. These lesions when untreated form a sinus or fistulous tract through the alveolar bone existing into the surrounding soft tissue. $^{7}$ The formation and location of sinus tract in endodontic infections are determined by two factors: the orientation of the root apex and the relationship of the site of perforation to muscle attachments on the maxilla and mandible particularly buccinator and mylohyoid muscles. ${ }^{6}$

The majority of odontogenic sinus tract have buccal/labial opening, because the palatal/lingual bone is more compact than the labial/buccal bone. Cases have been reported with extraoral sinus tract related to odontogenic infection. Cohen et al reported a case of cutaneous sinus tract along the inferior border of the mandible associated with mandibular first bicuspid. ${ }^{6}$ Mortensen et al, reported that out of 1600 teeth with periapical lesion, 136 teeth had sinus tract and 7 of these were draining extraorally. ${ }^{8}$ Gupta et al examined 160 teeth with preoperative status of periradicular inflammation and found that $18.1 \%$ had an odontogenic sinus tract. ${ }^{9}$ Baumgartner et al stated that, histological examination of 30 sinus tract 10 was found to have either complete or interrupted epithelial lining to the periapical lesion. ${ }^{3}$

In literature, three case reports have been reported with nasal fistula following intrusive luxation of the maxillary central incisor, ${ }^{10-12}$ out of which one case was in relation to a primary tooth. ${ }^{12}$ The occurrence of nasal sinus tract in these cases may be related to the closer proximity of apical foramen to the nasal floor as a result of intrusion.

However, in the present case, patient presented with a deformity of the oro-nasal complex resulting in close proximation of the central incisor to the nasal floor. This is an unusual presentation for an extra-oral sinus opening. These types of deformities are also seen in case of cleft lip and cleft palate. The distance from the maxillary root apices and the nasal floor is often short in these cases which may lead to uncommon sinus drainage sites such as the nasal cavity. ${ }^{13,14}$ All the previous cases were associated with trauma followed by periapical lesion with an extraoral sinus tract; sinus tract healed following conventional root canal treatment. ${ }^{10,11}$

Surgical intervention with de-epithelization of sinus was needed in this case due to the location and the chronicity of the lesion. The sinus tract can serve as a source of infection and comprehensive debridement cannot be achieved by conventional root canal treatment. Thus deepithelization of the sinus opening and primary closure of sinus opening surgically improves the prognosis of the treatment. ${ }^{15}$

The failure of non-surgical management in this is attributed to the cross contamination with microorganisms from the nasal floor. The nasal floor/ anterior nares are common site for colonizers/microorganisms like staphylococcus aureus. A sinus opening at this region creates a risk for the lesion to become contaminated with multidrug resistant organisms. ${ }^{16,17}$ The risk of carrying infection into central nervous system cannot be ruled out. This increases the chances for cavernous sinus thrombosis which has life threatening complications. ${ }^{18}$

In the present case, Considering the limited resources available in an institution based set up IRM was used as a root filling material, studies have reported good healing and success rate with both MTA and IRM as a root end filing material. ${ }^{19,20}$ Chong et al, suggested that the failure of the periapical surgery was more due to incomplete elimination of the primary source of infection and not related to the choice of root end filling material. ${ }^{21}$ However, tricalcium based silicate should be the preferred material for root end fillings.

\section{Conclusion}

The unique presentation of a nasal sinus tract opening as a result of odontogenic infection of tooth \#9 is reported. Anatomical deformities of the oro-nasal region could result in unconventional symptoms of dental infection. In this case, surgical de-epithialisation was necessary for complete resolution of the focus of infection.

\section{Source of Funding}

None.

\section{Conflict of Interest}

None.

\section{References}

1. Anderson NP. Persistent sinus tract of dental origin. Arch Dermatol Syphilo 1937;35:1062-73.

2. Baumgartner JC, Picket AB, Muller JT. Microscopic examination of oral sinus tracts and their associated periapical lesions. J Endod 1984;10(4):146-52.

3. McWalter GM, Alexander JB, del Rio CE, Knott JW. Cutaneous sinus tracts of dental etiology. Oral Surg 1988;66:608-14.

4. Cantatore JL, Klein PA, Lieblich LM. Cutaneous dental sinus tract, a common misdiagnosis: a case report and review of the literature. Cutis 2002;70(5):264-7.

5. Patni PM, Jain P, Prashanth MB, Jain N, Patni MJ. Nonsurgical management of an extraoral cutaneous sinus tract of odontogenic origin: an endodontic challenge. ENDO (LondEngl) 2010;4(4):285-91.

6. Cohen S, Burns RC. Pathways of the pulp. 7th ed. St. Louis: Mosby, Inc., 1998;30, 36.

7. Neelima A.M. Text book of Oral and Maxillofacial Surgery. 4th ed. Jaypee publications: India., 2016;840. 
8. Mortensen H, Winther JE, Birn H. Periapical granulomas and cysts. Scand J Dent Res 1970;78:241-50.

9. Gupta R, Hasselgren G. Prevalence of odontogenic sinus tracts in patients referred for endodontic therapy. $\mathrm{J}$ Endod 2003;29(12):798-800.

10. Fowler EB, Breault LG, Galvan DA. Nasal fistula associated with dental infection: a report of a case. $J$ Endod 2000;26(6):374-6.

11. Brickman JH, Kuchmas J, Skiribner JE. Nasal-apical communication associated with a pulpless tooth. $J$ Endod 1988;14(2):98-100.

12. Cobourne MT, Bloom KL, Harley KE, Porter SR. An unusual dental cause of nasal discharge: a case report. Int J Paediatr Dent 1996;6(3):187-9.

13. Jepsen O, Thomsen KA. Oere-, naese-, mund- og halssygdomme. Copenhagen: Munksgaard, 1970;209.

14. Valderhaug J. Reaction of mucous membranes of the maxillary sinus and the nasal cavity to experimental periapical inflammation in monkeys. Int J Oral Surg 1973;2,107-14.

15. Vire DE. Stalker WH. Kessler HP. Epithelium-lined oral sinus tract. Oral Surg 1982;53:209.

16. Frank DN, Feazel LM, Bessesen MT, Price CS, Janoff EN, Pace NR. The human nasal microbiota and Staphylococcus aureus carriage. PLoS One 2010;17;5(5).
17. Lina G, Boutite F, Tristan A, Bes M, Etienne J, Vandenesch F et al. Bacterial competition for human nasal cavity colonization: role of Staphylococcal agr alleles. Appl Environ Microbiol 2003;69(1):18-23.

18. Dixon OJ. The pathologic examination of cyst. JAMA 1926.

19. Chong BS, Ford TR, Hudson MB. A prospective clinical study of Mineral Trioxide Aggregate and IRM when used as root-end filling materials in endodontic surgery. Int Endod $J$ 2003;36:520-6.

20. Lindeboom JA, Frenken JW, Kroon FH, van der Akker HP. A comparative prospective randomized clinical study of MTA and IRM as root-end filling materials in single-rooted teeth in endodontic surgery. Oral Surg Oral Med Oral Pathol Oral Radiol Endod 2005;100:495-500.

21. Chong BS, Pitt Ford TR, Kariyawasam SP. Tissue response to potential root-end filling materials in infected root canals. Int Endod J 1997a;102-14.

How to cite this article: Ranjithkumar S, Krithikadatta J, Velmurugan N, Sreekumar K. Inter disciplinary approach of chronic periapical lesion with extraoral sinus tract opening on to the nasal floor: A case report. Indian J Conserv Endod2019;4(3): 